\title{
Alternating atrial electromechanical dissociation as contributing factor for pulsus alternans
}

\author{
FREEK W A VERHEUGT, HANA SCHECK, RICHARD S MELTZER, IOS ROELANDT \\ From the Noninvasive Laboratory, the Thoraxcentre, University Hospital Dijkzigt, Erasmus University, Rotterdam, The \\ Netherlands
}

SUMMARY Ten patients with mechanical pulsus alternans were studied by echocardiography and mechanocardiography. All had been or were in congestive heart failure. An atrial mechanism for pulsus alternans could be identified in two patients: one with primary congestive cardiomyopathy and one after aortic valve replacement for calcific aortic stenosis. Each strong systole was preceded by an "a" wave, while each weak systole was not. This was documented on both the apexcardiogram and the $M$-mode echocardiogram. Since both patients were in normal sinus rhythm with regular PP intervals, it was concluded that alternating atrial electromechanical dissociation was either the underlying mechanism or contributed to the pulsus alternans.

Thus, alternating atrial electromechanical dissociation exists and may cause pulsus alternans. Pulsus alternans is not necessarily the result of left ventricular myocardial dysfunction alone.

Pulsus alternans, originally described by Traube ${ }^{1}$ in 1872 , consists of an alternation of strong and weak ventricular contractions at regular intervals. In most cases, it is clinically recognised by palpation of peripheral arteries. It is never seen in subjects without heart disease and is usually associated with moderate to severe cardiac failure.

Several theories have been postulated to explain pulsus alternans including alternating contractile failure of some myocardial segments, ${ }^{2}$ incomplete metabolic recovery, ${ }^{3}$ and alternating high and low filling pressures of the left ventricle. ${ }^{45}$

We noted an atrial mechanism for pulsus alternans in one patient, and subsequently retrospectively studied a series of patients with pulsus alternans using external pulse recordings to see if this mechanism occurred in others as well.

\section{Patients and methods}

Ten patients with pulsus alternans were identified in the Thoraxcentre between 1974 and 1981, using external pulse recordings. All patients underwent $M$ mode echocardiography and phonomechanocardiography. The clinical data of the patients are listed in the Table.

Table Characteristics of patients

\begin{tabular}{cccl}
\hline \multicolumn{2}{c}{ Case No. Sex Age $(y)$} & Clinical state \\
\hline 1 & M & 24 & Congestive cardiomyopathy \\
2 & M & 56 & Two months after aortic valve replacement \\
3 & F & 60 & Six years after aortic valve replacement \\
4 & M & 62 & One month after aortic valve replacement \\
5 & M & 34 & Coronary artery disease, congestive heart failure \\
6 & M & 26 & Ventricular septal defect, Eisenmenger's \\
7 & M & 63 & One month after aortic valve replacement \\
8 & M & 42 & Congestive cardiomyopathy \\
9 & M & 33 & One month after aortic valve replacement \\
10 & F & 66 & Coronary artery disease, congestive heart failure \\
\hline
\end{tabular}

\section{Results}

In the presenting patient as well as in a second patient, alternating absence of the " $a$ " wave in both the apexcardiogram and M-mode echocardiogram was observed. Before each strong systole an "a" wave was present on both recordings and before each weak systole the " $a$ " wave was absent. In the other patients this abnormality was not seen.

Fig. 1 shows the simultaneous recording of the electrocardiogram, apexcardiogram, and phonocardiogram of case 1 . Note the alternating presence of atrial activity in relation to pulsus alternans visible in the apexcardiogram. In Fig. 2 the M-mode echocardiogram shows the same alternating absence of the "a" wave on the anterior mitral valve leaflet motion 


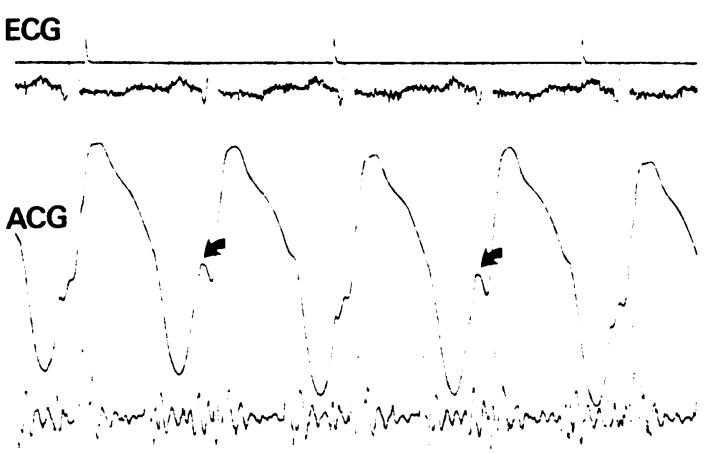

Phono

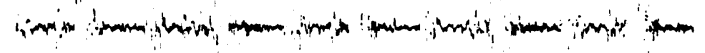

Fig. 1 Electrocardiogram (ECG), apexcardiogram $(A C G)$, and phonocardiogram (Phono) of case 1. Note that each strong systole is preceded by an " $a$ " wave (arrows) and the weak systoles are not.

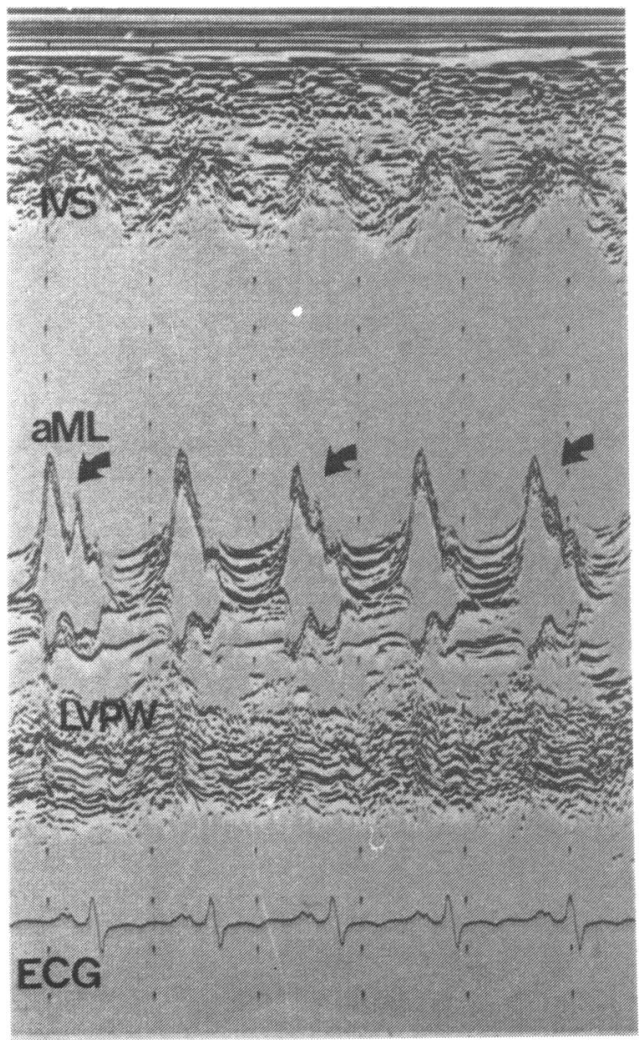

Fig. $2 M$-mode echocardiogram of case 1 . Note the alternating presence of the " $a$ " wave (arrows) in the anterior mitral valve leaflet $(a M L)$ motion. The left ventricular posterior wall ( $L V P W)$ amplitude and velocity of motion are higher after an " $a$ " wave. ECG, electrocardiogram; IVS, interventricular septum.
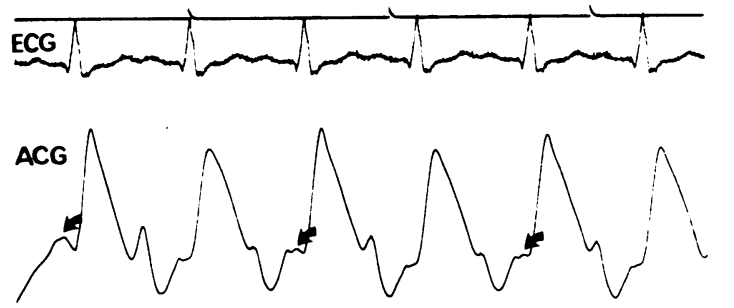

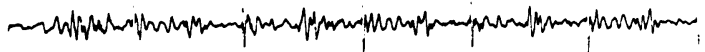
Phono

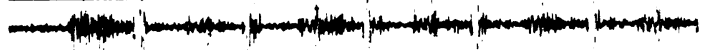

Fig. 3 Electrocardiogram (ECG), apexcardiogram (ACG), and phonocardiogram (Phono) of case 2. Note that each strong stysole is preceded by an " $a$ " wave (arrows) and the weak systoles are not. The electrocardiogram shows complete left bundle-branch block.

pattern. Alternating strong and weak left ventricular posterior wall contractions are present: both the amplitude of motion and endocardial velocity of anterior motion are alternating.

Fig. 3 and 4 show the recordings of case 2. In Fig. 3 the alternation of atrial mechanical activity is seen on the apexcardiogram and this was associated with pulsus alternans, as seen in Fig. 4 on the carotid pulse recording. Note that the "a" wave on alternate beats as seen in the anterior mitral valve leaflet tracing always precedes a strong beat on the carotid pulse recording.

Since both patients were in sinus rhythm with

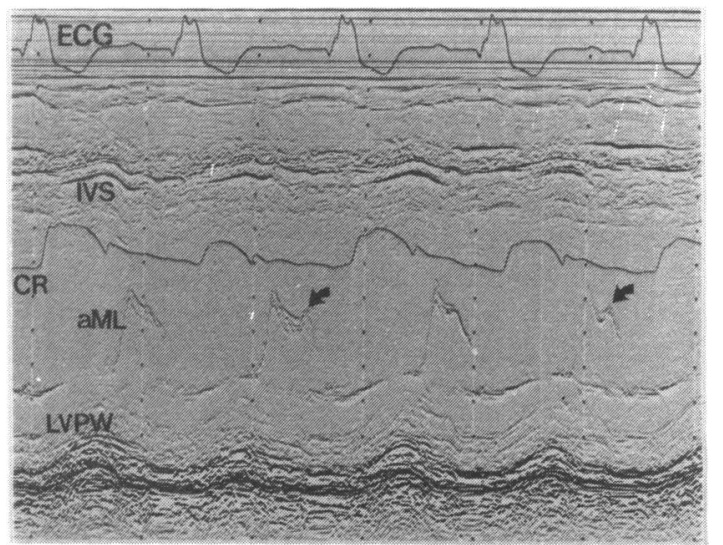

Fig. 4 M-mode echocardiogram of case 2 with simultaneous carotid pulse recording $(C R)$. The alternating presence of the " $a$ " wave on the anterior mitral valve leaflet $(a M L)$ motion pattern correlates well with the alternation in the carotid pulse. The motion of the interventricular septum (IVS) is paradoxical (probably the result of operation). The electrocardiogram shows complete left bundle-branch block. ECG, electrocardiogram; LVPW, left ventricular posterior wall. 
regular PP intervals, the alternating absence of atrial contraction is caused by a 2:1 electromechanical dissociation and appears responsible for the pulsus alternans in these patients.

\section{Discussion}

Alternating atrial electromechanical dissociation is a previously undescribed mechanism. Presumably it causes alternating strong and weak filling of the ventricles and therefore pulsus alternans.

Continuous atrial electromechanical dissociation has been reported after conversion of atrial flutter or fibrillation into sinus rhythm. ${ }^{6}$ Often this is only temporary, but sometimes it can be permanent ${ }^{7}$ when atrial fibrillation is longstanding. The mechanical failure of the atrium, in most cases the left atrium, is attributed to severe atrial muscle damage during the arrhythmia. Alternating atrial electromechanical dissociation may also be the result of atrial muscle damage. Left atrial enlargement seems to be a less important factor, since it occurred in both the patients with alternating atrial electromechanical dissociation and those without.

Pulsus alternans is usually associated with severe left-sided heart disease, such as severe coronary artery disease, aortic valve disease, and cardiomyopathy. Its exact cause is not understood, but it is perhaps caused by (1) alternating contractile failure of some myocardial segments, ${ }^{289}$ or (2) alternating filling of the ventricles. ${ }^{4} 10$ The most persuasive demonstration of the latter mechanism was by Veenendaal and Nanda, ${ }^{5}$ who studied a patient with a mitral valve prosthesis and pulsus alternans by echocardiography. They found that the echocardiogram showed alternating failure of prosthetic valve opening, with alternating filling of the left ventricle. The mechanism of pulsus alternans in the two patients illustrated here is probably related to that of Veenendaal's patient, whereas this mechanism played a minor role in the other eight patients in our series. The regular presence of the fourth heart sound in the patients showing alternating atrial electromechanical dissociation should be noted. In spite of pulsus alternans, its intensity did not vary on the phonographic recordings. Since the fourth heart sound is believed to represent rapid changes in late ventricular filling, the contribution of atrial contraction to the generation of this sound must be reconsidered, at least in patients with alternating atrial electromechanical dissociation. The constant fourth heart sound favours the theory of alternating contractile failure of some left ventricular myocardial segments as a cause of pulsus alternans. ${ }^{9}$

Thus, it is likely that pulsus alternans can be caused by a variety of mechanisms. Unfortunately, our series of patients with pulsus alternans is too small for definite conclusions to be made on the mechanism described in this report. Further studies may indicate more precisely its incidence and may provide further insight into its pathophysiology.

\section{References}

1 Traube L. Ein Fall van Pulsus Bigeminus. Berl Klin Wochenschr 1872; 9: 185.

2 Green HD. The nature of ventricular alternation resulting from reduced coronary blood flow. Am $\mathcal{F}$ Physiol 19351936; 114: 407-13.

3 Straub H. Dynamik des Herzalternans. Deutsches Archiv fur klinische Medizin 1917; 123: 403-34.

4 Mitchell JH, Sarnoff SJ, Sonnenblick EH. The dynamics of pulsus alternans: alternating end-diastolic fiber length as a causative factor. $\mathcal{F}$ Clin Invest $1963 ; 42$ : 55-63.

5 Veenendaal $M$, Nanda NC. Noninvasive diagnosis of mitral prosthesis malfunction. Am $\mathcal{J}$ Med 1980; 69: 45862.

6 Ikram H, Nixon PGF, Arcan T. Left atrial function after electrical conversion to sinus rhythm. Br Heart f 1968; 30: 80-3.

7 Olsson SB, Orndahl G, Erneström S, et al. Spontaneous reversion from long-lasting atrial fibrillation to sinus rhythm. Acta Med Scand 1980; 207: 5-20.

8 Noble RJ, Nutter DO. The demonstration of alternating contractile state in pulsus alternans. F Clin Invest 1970; 49: 1166-77.

9 Gentzler RD, Gault JH, Liedtke AJ. Pulsus alternans (PA) in man without alternation of end-diastolic volume (EDV) (abstract). Circulation 1972; 46, suppl II: 155.

10 Gleason WL, Braunwald E. Studies on Starling's law of the heart. VI. Relationships between left ventricular enddiastolic volume and stroke volume in man with observations on the mechanism of pulsus alternans. Circulation 1962; 25: 841-8.

Requests for reprints to Dr J Roelandt, Thoraxcentre, Erasmus University, Postbox 1738, 300 DR Rotterdam, The Netherlands. 